\title{
Dynamic simulation and curve fitting of folding furniture based on geometric analysis and MATLAB
}

\author{
Wenqi Zhang ${ }^{1, a}$ \\ Electrical engineering and automation, North China Electric Power University, \\ Hebei, Baoding, 071000,China \\ corresponding author: 18331130993@163.com
}

Key words : folding table, curve fitting, geometric stress analysis

Abstract

According to the problem that the current residential space tension, more and more folding furniture are produced. In this paper, the dynamic equation of the folding table is obtained by using geometric knowledge and nonlinear constrained optimization model, and it illustrates the curve fitting model of folding table in dynamic process.

\section{Introduction}

At present, the tension of residential space leads to the emergence of more and more folding furniture. In this paper, we use geometric knowledge and nonlinear constrained optimization model to obtain the description equation of the dynamic process of folding table.

According to the structure before and after the table is symmetric, we only in table $1 / 4$ as the research object, using space geometry knowledge, a geometric model is derived. And then using MATLAB software programming, draw the changes during the folding table dynamic map. Then find out the folding table for each wood desktop relative angle of each wood the length of slot length data of each batten, related results are shown in Table 1 and then establish the corresponding three-dimensional coordinates, calculate the table angle of each endpoint coordinates, draw the corner of the table edge line graph, and use the MATLAB toolbox for fitting the calculated function between the corner of the table edge, and the fitting effect is done Analysis. 


\begin{tabular}{c|c} 
Defination & Significance \\
\hline Symbol & Batten width $(\mathrm{cm})$ \\
\hline $\mathrm{D}$ & Seam width \\
\hline$\Delta x$ & Board length $(\mathrm{cm})$ \\
\hline $\mathrm{L}$ & Board width $(\mathrm{cm})$ \\
\hline $\mathrm{W}$ & The first $\mathrm{n}$ bar \\
\hline $\mathrm{N}$ & Number of batten \\
\hline $\mathrm{T}$ & The length of the first batten from outside $(\mathrm{cm})$ \\
\hline$l_{1}$ & The length of the $\mathrm{N}$ batten from outside $(\mathrm{cm})$ \\
\hline$l_{n}$ & Table height $(\mathrm{cm})$ \\
\hline $\mathbf{H}$ & Table radius $(\mathrm{cm})$ \\
\hline $\mathrm{R}$ &
\end{tabular}

Due to the folding in different size, we can describe in a time specific forms of the folding table, but we can describe the dynamic changes of the folding table with each stick angle changes. First of all, we know that the folding table around the symmetry, we can use geometric knowledge for change 1/4 angles. Finally, according to the initial time and the final form of the two states for the leg of the table wooden slotted length.

\section{Design of the model}

We make the two longest legs of the folding table (on the same side of the board when it is not folded)

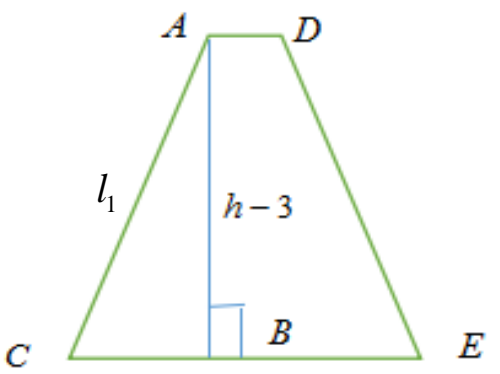

(The A point is the distance from one end of the longest piece of wood to the horizontal plane, and the distance between the A point and the horizontal surface is reduced by $3 \mathrm{~cm}$ due to the actual height of the table, including the thickness of the desktop 3cm)

$$
l_{1}+A D+D E=L=120 \mathrm{~cm}
$$




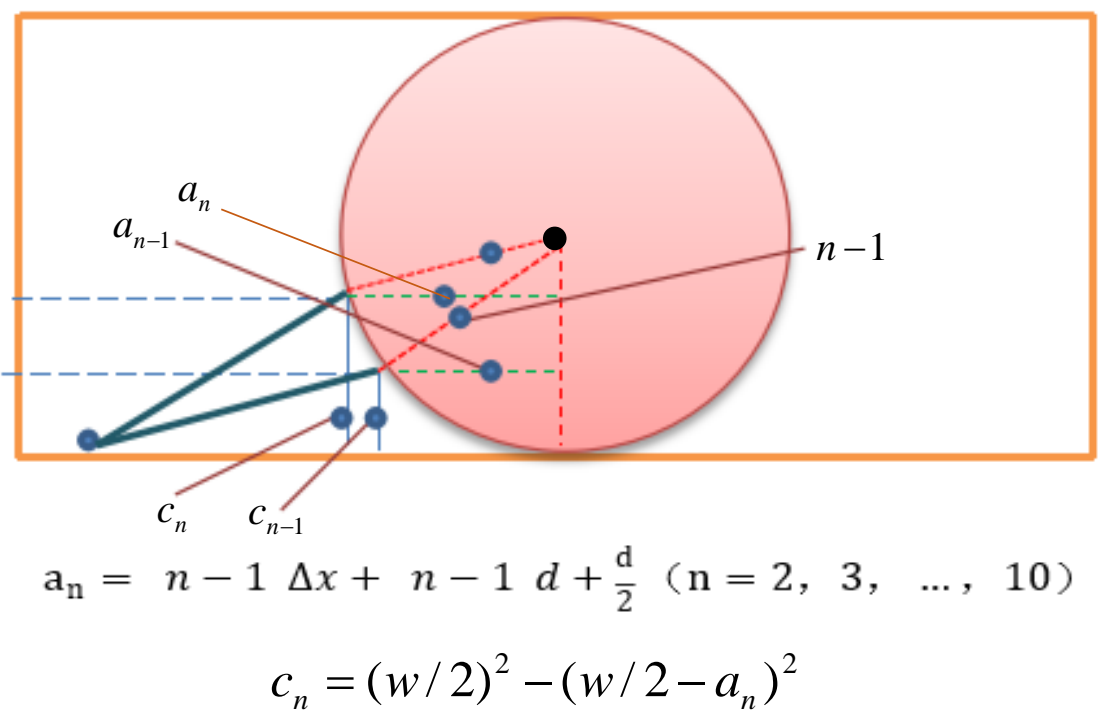

In order to solve the rotation angle of the wood, we make a sketch map of the folding stool.

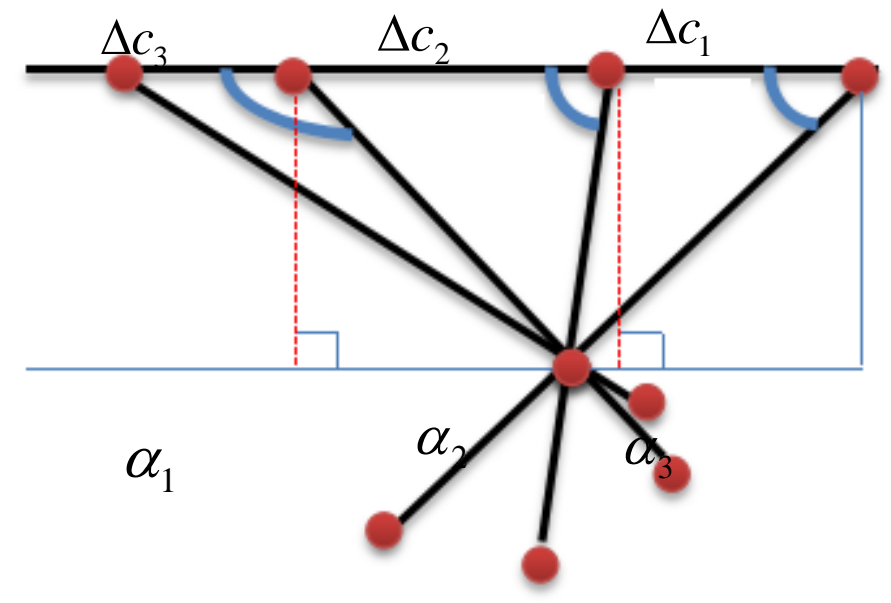

Dynamic change process: due to the size of the force is unknown, the relationship between folding table and time can not be determined, we can only determine the table from the plate to the fold after the completion of this process, any angle of the corner of the table position, for example when the long wooden turned 60 degrees, 65 degrees, 70 degrees, through the program can get the relative wooden desktop the rotation angle, as shown in table

\begin{tabular}{c|c|c|c}
\hline & Angle of 60 degrees & Angle of 65degrees & Angle of 70degrees \\
\hline First root & 60 & 65 & 70 \\
\hline Second root & 71.5106 & 78.8219 & 82.0272 \\
\hline Third root & 79.728 & 84.9829 & 90.063 \\
\hline Fourth root & 85.977 & 91.0414 & 95.8979 \\
\hline Fifth root & 90.7653 & 95.6054 & 100.2279 \\
\hline Sixth root & 94.3857 & 99.0138 & 103.1289 \\
\hline Seventh root & 97.0154 & 101.5264 & 105.7514 \\
\hline Eighth root & 92.5856 & 103.2894 & 107.5541 \\
\hline Ninth root & 99.8755 & 104.5681 & 108.5697 \\
\hline
\end{tabular}


According to the above model, the use of MATLAB software, programming to calculate the length of each piece of wood, rotation angle, the length of the long slot as shown in table

\begin{tabular}{c|c|c|c|c|c|c|c|c|c}
\hline & First & Second & Third & Fourth & Sixth & Sixth & Sevenh & Eighth & Ninth \\
\hline Slot length & 0 & 4.5018 & 7.9340 & 10.73 & 12.994 & 14.793 & 16.164 & 17.128 & 17.892 \\
\hline Batten length & 50.09 & 46.632 & 63.154 & 40.65 & 38.568 & 37.336 & 36.214 & 35.625 & 35.14 \\
\hline Rotation angle & 73.89 & 85.33 & 93.254 & 99.36 & 101.56 & 106.55 & 108.57 & 110.25 & 111.32 \\
\hline
\end{tabular}

Based on the above analysis, the MATLAB program is used to draw the edge of the table.

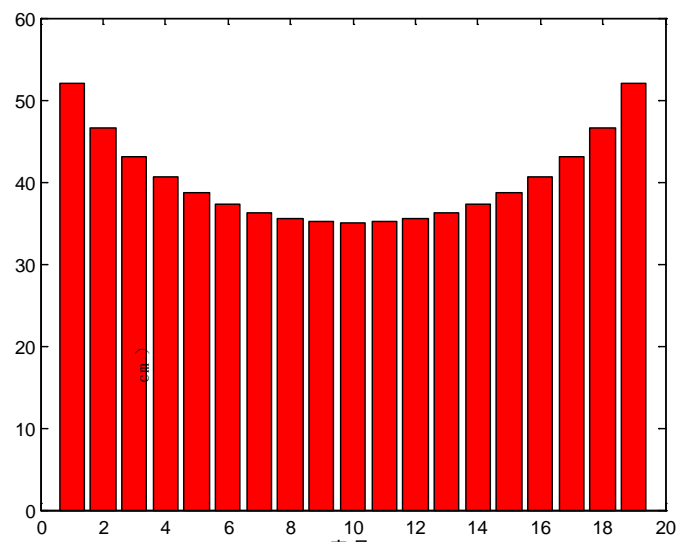

Batten diagram

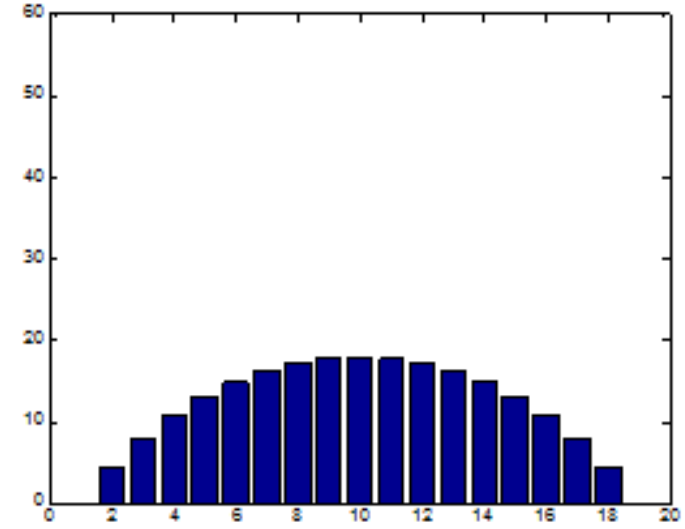

Slotted length column
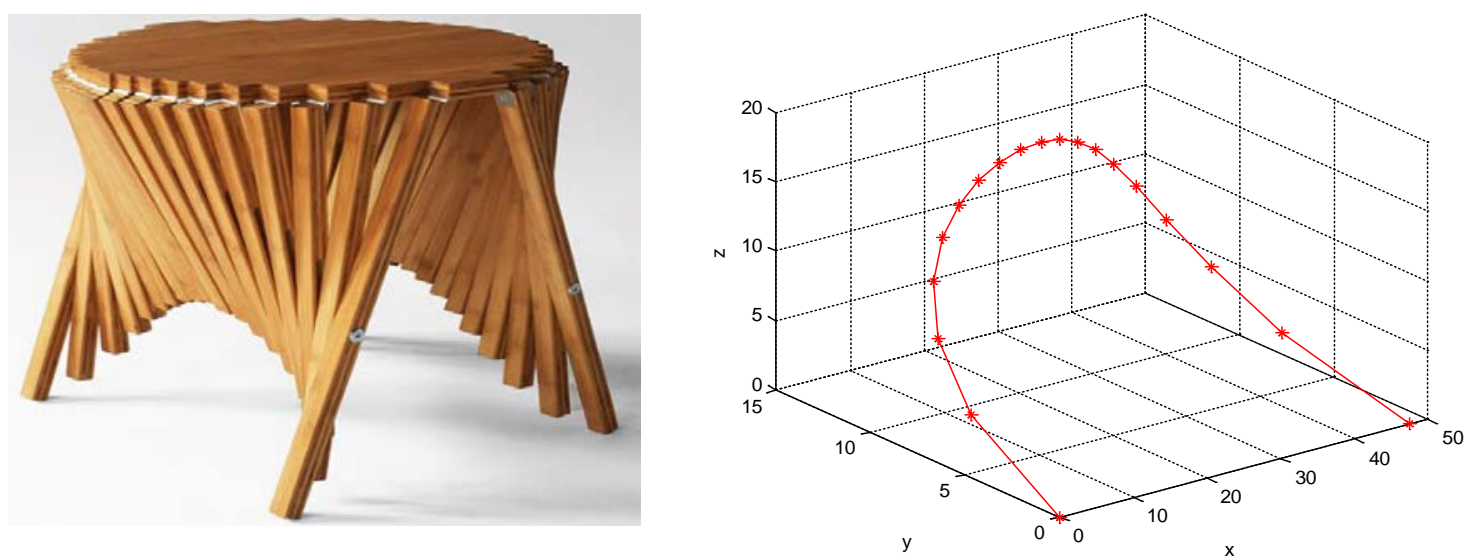

Based on the above analysis, the use of MATLAB programming, drawing the edge of the table edge line, as shown in Figure.

\section{Conclusion}

Based on the geometric stress analysis to simulate the stress model of the folding table, according to the analysis, we get the relationship between wood length, slot length and rotation angle. In addition, the application of MATLAB software to fit the 
curve edge folding table in the dynamic process, provides effective parameters for processing and production of folding tables and chairs.

\section{Reference}

[1] The application of MATLAB in the analysis of experimental data and curve fitting in, Dongsheng Yang.

[2] Curve fitting based on curve fitting toolbox,Qingwan $\mathrm{Hu}$.

[3] The research and design of TRIZ based on the theory of folding furniture,Jiaxin Lin,Guiping Nie

[4] Flat tensegrity system and geometric stress analysis,Yaozhi Luo,Shilin Dong.

[5] MATLAB to solve the problem of higher mathematics, Beijing: Tsinghua University press, 2008, Dingyu Xue,Yangquan Chen.

[6] Mathematical modeling, Beijing: Mechanical Industry Press, 2009.8.[7] Qiyuan Jiang,Qixuan Ye. 